\title{
What is a Visual Novel?
}

\author{
JANELYNN CAMINGUE, University of California, Santa Cruz, USA \\ ELIN CARSTENSDOTTIR, University of California, Santa Cruz, USA \\ EDWARD F. MELCER, University of California, Santa Cruz, USA
}

\begin{abstract}
Visual Novel (VN) is a widely recognizable genre of narrative-focused games that has grown in popularity over the past decade. Surprisingly, despite being so widely recognizable, there is not a singular definition to help guide the design and analysis of such games-with academic definitions and implementations ranging from "interactive textbooks" to "adventure games with multi-ending stories". In this paper, we present a unified definition of VNs drawn from an analysis of 30 prior academic definitions. We also examined 54 existing VNs to further refine our definition and employ a deeper analysis of the interactivity within VNs. We highlight key features of VNs that arise in our definition, and discuss resulting implications for the design of VNs. This work is relevant for narrative game designers and researchers, affording a more unified structure and clearer guidelines to identify, analyze, and design future $\mathrm{VN}$ games.
\end{abstract}

\section{CCS Concepts: • Human-centered computing $\rightarrow$ Empirical studies in HCI.}

Additional Key Words and Phrases: Visual Novel, interactive narrative, meta-analysis, video games

\section{ACM Reference Format:}

Janelynn Camingue, Elin Carstensdottir, and Edward F. Melcer. 2021. What is a Visual Novel?. Proc. ACM Hum.-Comput. Interact. 5, CHI PLAY, Article 285 (September 2021), 18 pages. https://doi.org/10.1145/3474712

\section{INTRODUCTION}

The Visual Novel (VN) is a widely popular and recognizable narrative-focused game genre that originated in Japan [12, 28, 67, 77]. Their popularity has been steadily increasing outside of Japan with 2,272 VNs currently available for purchase on Steam within the $\mathrm{US}^{1}$, and 27,140 VNs archived on community run websites such as $\mathrm{VNDB}^{2}$ that aim to document the production of VNs. This popularity has been bolstered further still with the increase of free VN game engines such as Ren'Py [18]. VN engines like Ren'Py provide a simple scripting language and easy to use graphical user interface, which have made the genre accessible to designers and authors without technical backgrounds. This in turn has made VNs simple to construct and beginner friendly for novice designers, while simultaneously allowing expert designers to produce complex and engaging work. Furthermore, the overall ease of creation, distribution, and accessibility for players at a variety of skill levels with respect to VNs has also led to their frequent use in academic research-most commonly in high-impact domains such as education $[4,13,26,29,31,40,49,51,68,87]$ and health

\footnotetext{
${ }^{1}$ Number collected on April 18th, 2020

${ }^{2}$ The Visual Novel Database - https://vndb.org, number collected on April 18th, 2020
}

Authors' addresses: Janelynn Camingue, University of California, Santa Cruz, USA, 1156 High St, Santa Cruz, CA, 95064, jcamingu@ucsc.edu; Elin Carstensdottir, University of California, Santa Cruz, USA, 1156 High St, Santa Cruz, CA, 95064, ecarsten@ucsc.edu; Edward F. Melcer, University of California, Santa Cruz, USA, 1156 High St, Santa Cruz, CA, 95064, eddie.melcer@ucsc.edu.

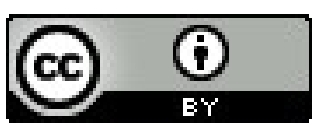

This work is licensed under a Creative Commons Attribution International 4.0 License.

(c) 2021 Copyright held by the owner/author(s).

2573-0142/2021/9-ART285. https://doi.org/10.1145/3474712

Proc. ACM Hum.-Comput. Interact., Vol. 5, No. CHI PLAY, Article 285. Publication date: September 2021. 
[77, 93]. This highlights the significance of VNs as an area of study in their own right, and the value that improving understanding of such designs can provide.

Unsurprisingly, as a result of the popularity of VNs both commercially and within research, there have been a number of definitions created to provide a high-level description of key design features underlying the genre. However, these definitions can vary wildly, which in turn has substantial implications for the design of such games. For instance, definitions have included "VNs are interactive textbooks" [87], which is in substantial contrast to "Adventure Games born in Japan, that makes use of attractive characters, narrative engagement, puzzles and other interactive features to maintain user interest while submerging players in complex stories" [77]. This wide range of definitions can prove problematic for both designers and researchers of VNs, providing notably different (under and over constrained) interpretations of the potential design space for such games.

Despite this lack of consensus on a clear definition of VNs as a genre, they are still easily identifiable as such by the publishers who produce them and the community of players who enjoy them. Examples of this consensus can be seen through fan community documentation efforts on $\mathrm{VNDB}^{2}$ as well as the community genre labels on Steam. Furthermore, IndieDB ${ }^{3}$ (a popular database for indie games) has a specific genre section for Visual Novel, as well as Twitch ${ }^{4}$ (the largest platform for streaming games) which also has a tag for Visual Novel. This indicates that there is a set of very specific criteria that can be used to identify VNs, which also implies that a more unified definition and formalized understanding of VNs is achievable.

While there is evidence of an implicit understanding of VNs illustrated above, there is no clearly defined census on the set of features that define them-as exemplified by the 2,556 tags used to describe features of VNs on VNDB ${ }^{5}$. Furthermore, these tags pertain to both the content and structure of VNs. A lack of clear definition impacts the ability of designers to create VNs and realize the full potential design space of the genre, and similarly makes classifying and analysing VN games difficult for researchers. Ultimately, creating a unified definition of VNs as a genre as well as understanding the key elements of a VN's design allows us to better inform future work in the area of VNs and interactive narrative.

In this paper, we propose a unified definition of VNs developed from analyzing existing definitions of VNs as a genre and playing through a corpus of $54 \mathrm{VNs}$ to refine the analysis. We then discuss key features from our unified definition and their implications for the enhanced design of future VNs.

\section{RELATED WORKS}

\subsection{Game Genre Studies}

Game genres are conceptual tools used to understand games and their contexts [20, 21, 33, 83]. While not the only tool to classify games $[3,8,20,24]$, genre is arguably the most widely used manner to describe, group, and contrast games based on their defining characteristics. For games, genre is commonly conceptualized, and sometimes named after, specific sets of mechanics, control schemas, player goals, narrative structure and more, e.g., Metroidvania, Walking Simulators, and Role-Playing Games (RPGs). As an illustrative example, the well established first-person shooter (FPS) genre broadly consists of first-person camera perspective, where the core gameplay consists of the player pointing and clicking to a location where a projectile or effect is meant to make an impact of some description, often requiring movement of the player's camera perspective through

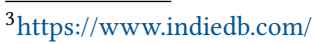

${ }^{4}$ https://www.twitch.tv/

${ }^{5}$ The Visual Novel Database - https://vndb.org, number collected on April 18th, 2020
} 
a virtual space. FPS games most often present this interaction via an entity carrying and using a gun, hence the name.

Game genre not only allows us to classify and describe games, but provides a common ground to contextualize and understand contributions to game design broadly as a research field [33]. This makes the study of genre and its construction of great importance for the game research community as a whole.

Construction of genre has been widely discussed in academic literature [3, 8, 20, 21, 33, 38, 55], as well as in commercial and consumer communities. Within the context of commercial game genre definitions, there is a tendency to rely too heavily on the representational characteristics (i.e., visual aesthetics) of a game [8]. In contrast, game researchers have argued that games and their genres should also be i) described with respect to their interactivity and how a player nontrivially progresses through a game [8]; ii) described through their game architecture in the form of high-level design patterns [54]; or should be iii) described in terms of a simulation since knowledge and experience-and by extension the story-is created through player actions [2]. With respect to academic explorations, this more detailed analysis has been done in a number of past works categorizing and defining games and their genres as a whole [3, 27, 91]. These game genres have included idle games [5], educational games [61, 63], serious games [56], games for rehabilitation [74], games for dementia [19,59], exertion games [65], affective games [52], and games and simulations [48].

There are a number of approaches to the construction of game genres. These include formulation of guidelines [21,33], development of models [38, 83], and using both quantitative [55] and qualitative methods [6] to study existing games and meta-data associated with them. Building on this related work, we employ a mixed methods approach, utilizing meta-data like Steam tags to identify what the broader community considers to be a part of the VN genre as suggested by Li and Zhang [55], and utilizing an extensive review of the literature--similar to [38] to identify which features and design elements existing work used to define and refer to the genre,. Both of these techniques are used in tandem to guide the analysis of existing and commercially available games.

\subsection{The Visual Novel Genre}

Visual Novels (VNs) are a highly popular genre of interactive narrative games. For instance, VNs have been released on a large range of platforms such as PC [76], mobile [88], and PS Vita [69]. The VN player community has also created VNDB, a community managed database for tagging and reviewing VNs with over 27,000 entries as of April 18th, 2020. Furthermore, commercial VNs have also seen recent interest as a subject of study in academic papers. E.g., Carstensdottir et al. [15], examined the structure and narrative progression of various interactive narrative games including VNs such as Doki Doki Literature Club and Long Live the Queen. Similarly, other popular commercial VNs such as Hatoful Boyfriend have been the subject of work analyzing how the VN subverts tropes and conventions of Japanese popular culture [30].

Despite relatively simple core mechanics, VNs have incorporated a variety of themes ranging from Horror Survival (e.g., Spirit Hunter: Death Mark [46]) and Murder Mystery (e.g., Danganronpa V3: Killing Harmony [82]) to Historical Fantasy (e.g., Hakuoki: Edo Blossoms [42]) and Romance (e.g., Amnesia: Memories [41]). The increasing popularity of VNs, coupled with the introduction of free and accessible VN authoring tools, has raised both interest and awareness for the use of VNs in other applications such as education [13]. However, the question of how to define VNs as a genre has remained elusive.

The genre's heavy use of text and emphasis on reading as a central activity has prompted repeated comparisons to books and seen VNs referred to as their interactive counterparts, such as in [87] and [10]. Notably, some literary genres have made a successful transition between the two narrative 
mediums, with both straight and queer players recognizing romance VNs as "romance novels in interactive visual form" [60]. However, while similar, VNs remain distinct from their non-digital and non-interactive counterparts for several reasons. First, VNs only show one snippet of text at a time as the player interacts with the game [31], the snippet being dependent on player action or choices and even shaping future interaction. Further, while VNs share many traits with choose-your-own adventure books [10] - a legacy shared with most modern interactive fiction and storytelling design in video games $[34,62,64]-V N s$ often incorporate more involved mechanical and constraint based gameplay to impact the progression of the story and the relevance/complexity of player choice [15].

As a whole, the VN genre has ultimately not been extensively explored and researched [12]. While VNs have had a number of definitions in academic and commercial literature, most of these definitions have focused primarily on the aesthetics of the games [11, 16, 26, 28, 29, 31, 40, 49, 57, 66$68,70,71,75,79]$, structure and choice $[7,10,22,28,29,36,57,67,71,71,80]$, and story quality $[12,16,49,53,79]$. Surprisingly, there has been a limited focus on interactivity within existing VN definitions, primarily referring to interactivity as being minimal or lacking [4, 26, 36, 40, 67]. Notably, there is some disagreement to what extent story quality and other elements like interaction influence how VNs should be defined. For example, it has been argued that VNs are "reliant on the text and the actual content instead of interactivity" [12], while others describe certain types of input performance and interactivity-such as the classic click-to-continue interaction where the player clicks to receive the next part of story-as being a part of VNs [7, 36, 40, 57].

More directly related to the work presented in this paper, Lynch et al. [58] focused on a broader understanding of VNs where they examined six existing VNs and their feature sets-three of which were in the top 100 most popular games on VNDB. Although it did provide a wider perspective of VNs, this work was mainly focused on how a game engine could implement the features found in their data set and did not fully define features that were essential to all standard VNs. This work also did not consider or compare existing definitions of VNs. Instead, the focus was on the problem of how to make a $\mathrm{VN}$ and not what is a $\mathrm{VN}$.

Ultimately, most definitions of VNs as a genre only contain a small number of elements, leaving out concepts deemed as central to the genre in other definitions. A unified definition would allow for a more comprehensive view and approach to the study/design of VNs, as well as to the use of VNs in development of various high impact application areas, such as education and health, where their accessibility and ease of use has the potential to be leveraged with great effect.

\subsection{Serious Applications of Visual Novels}

VNs have been used extensively as a tool to address numerous serious topics such as education, health, and disabilities, to name a few. For instance, VNs have been employed to teach topics such as second-language acquisition [25, 86], chemistry [89, 92], life management [49], research skills [87], and cooking $[44,45]$. Recent work has even constructed a taxonomy categorizing how VNs can deliver educational content [13]-describing the different teaching strategies currently employed by educational VNs. With respect to health applications, past work has focused on teaching health concepts such as nutrition [77], explored how an individual VN can improve patient perspectives and support desired health outcomes [93], and addressed complex health related topics such as disability [17, 84].

Another frequent serious application of VNs is exploring narratives and issues faced by underrepresented communities/populations, such as gender inequality and queer narratives [90]. For instance, recent work by Salter et al. [79] has examined the design of Butterfly Soup and its portrayal of queer identity and experience. Schaufert [81] has similarly analyzed the design of Dream Daddy, highlighting that it normalizes the "queer daddy figure" yet can create negative feelings as well.

Proc. ACM Hum.-Comput. Interact., Vol. 5, No. CHI PLAY, Article 285. Publication date: September 2021. 
Notably, Dream Daddy also became an exceptionally popular and successful VN-where in 2017, it replaced first-person shooter Overwatch "as the most-discussed videogame on Tumblr for the first time in more than nine months," and it was at the "top of Steam's global sales chart, unseating battle-royal phenomenon PlayerUnknown's Battlegrounds" [39]. The growing use of VNs as serious contexts highlights the potential impact VNs could have beyond entertainment, and underscores the importance of having a unified definition for $\mathrm{VN}$ in order to support research and design for serious applications.

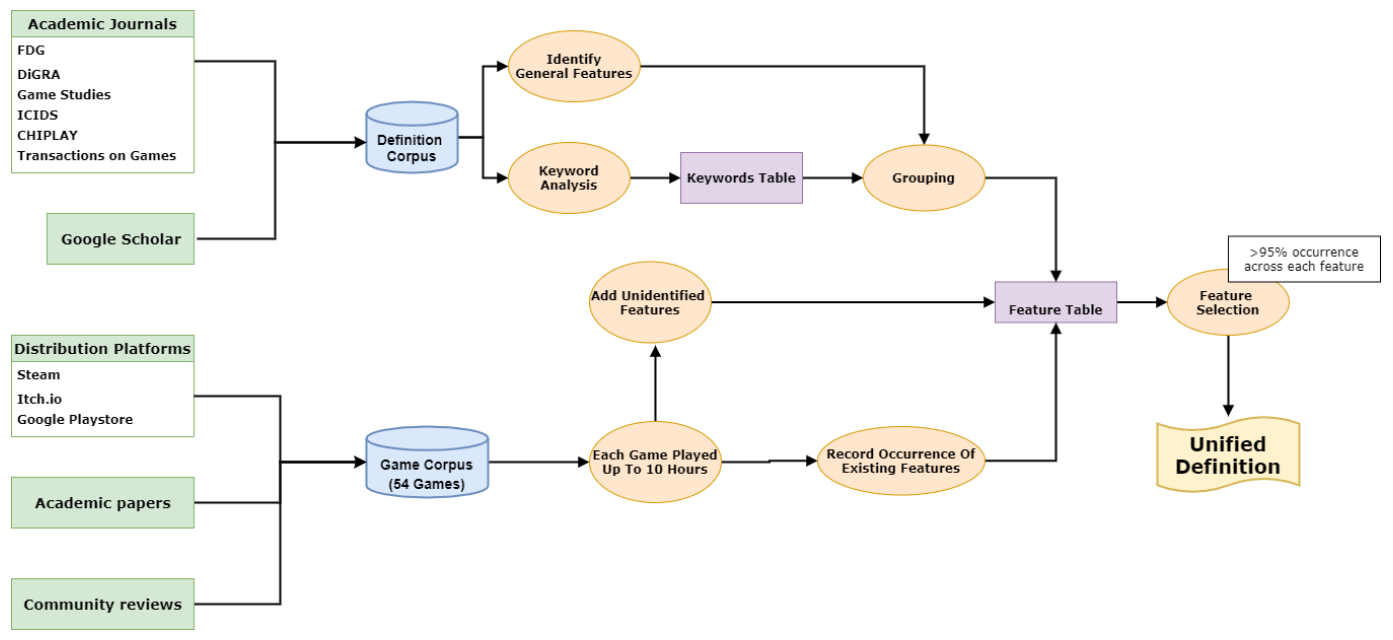

Fig. 1. An overview of the methodology used to construct a unified definition of VNs.

\section{METHODOLOGY}

Creating a unified definition of VNs requires identifying their essential core design features. To do this, we performed a comprehensive search for VN papers and definitions-resulting in the identification of 30 existing definitions from the literature. These definitions were then analyzed to identify key theoretical design features underlying VNs. Simultaneously, we also collected a $\mathrm{VN}$ corpus of 54 games in order to analyze playable instances of VNs in comparison with the theoretical definitions. Each VN was played or at least 10 hours or until finished, whichever came first, and common design features that emerged during gameplay but weren't identified in the theoretical design features were recorded. The VN corpus was then played through and labeled a second time utilizing all features from both the theoretical and emergent feature sets. The labeling using the larger set of design features was then analyzed, resulting in a set of core design features that occurred in $95 \%$ or more of the VN games from the corpus. See Figure 1 for a visual overview of the process. These core features were then used to construct a unified definition of VNs.

For feature labeling, we employed a single researcher approach. This was done to keep the data consistent across the study by avoiding the creation data inconsistencies caused by individual differences between researchers. Importantly, this approach has been employed successfully before in prior game research-specifically for analyzing narrative games [15] and educational visual novels [13]-and is therefore a reasonable method to employ for this kind of analytic research However, in order to mitigate individual researcher bias, the researcher that performed feature labeling also met weekly with two additional senior researchers to present, discuss, refine, and finalize all identified features and labeling. 


\subsection{Academic Definitions}

Collecting definitions. First, the researchers compiled academic papers that addressed the topic of VNs. These papers were identified through a systematic review of proceedings of recognized venues for technical games research, game studies, and interactive narrative-specifically FDG, DiGRA, ICIDS, CHIPLAY, Game Studies, and Transactions on Games. This was then augmented with a search through Google Scholar. The keyword "visual novel" was used across all searches, and papers that did not provide an explicit definition of a VN were excluded. All papers selected for the analysis were required to contain an explicit $\mathrm{VN}$ definition. This requirement was included to prevent accidental researcher bias from interpreting and/or extracting definitions from papers that didn't explicitly provide one. This process resulted in 30 papers, each containing a definition of a VN. Afterwards, a table was constructed for analysis. The rows consisted of the 30 definitions collected, and the columns consisted of all VN features identified in the various definitions-these features are explained in more detail in section 4.1. In one specific case, for the concept of level of interactivity, the researcher recorded the varying degrees of interactivity that were detailed in each definition-ranging from only pressing a button to advance the story (no interactivity) to providing choices that impact the story (moderate interactivity) or minigames and puzzles to complete (high interactivity). A first pass was done to find commonalities and differences between the general features that each definition covered. The result was then used to inform what specific features to identify on the first play through of the games.

To further reduce bias, the researcher performed a second pass, where they performed detailed review of each definition with two additional researchers. Another table was constructed to describe exact keywords for the identified VN features. One by one, the researchers reread each definition, highlighted the key words within that definition, and if that keyword was not already in the table, the keyword was added as a new column. Otherwise, if the keyword already existed within the table or a very close synonym existed, the researcher marked down that this definition contained this existing keyword. Notably, this approach helps to compare the explicit wordings of definitions without interpretation by the researchers. These keywords were also ultimately used to construct the final unified definition.

\subsection{VN Search Strategy}

In order to identify which features from academic definitions were also commonly found in VNs (and ultimately aid in constructing a more grounded unified definition), the researchers constructed a corpus of games. Notably, this approach of collecting and analyzing of a large corpus of related games has also previously been employed in other genre defining work, e.g., $[5,38,55]$. The researchers began their search by formulating a set of three selection criteria. First, the game is tagged as a $\mathrm{VN}$ on its respective distribution platform. These tags are usually assigned by publishers, developers, and in the case of Steam by players. Games that fulfill this criterion are representative of the industry and players themselves identify as VNs. The second criterion was that the game's published description, or an academic paper, explicitly identifies a particular game as a VN. This criterion helps filter for games that developers or academics consider VNs. The third criterion was that the game must be documented in VNDB, meaning they are considered VNs by the player community. The researchers collected the initial set of VNs from previous academic literature that explicitly identified VNs. These games fulfilled the second selection criterion. The researchers then collected an additional sample based on critical acclaim and popularity within the VN community. The researchers then looked for VNs that fulfilled one of the three selection criteria on three different distribution platforms. These platforms were Steam, Itch.io, and the Google Play Store. The researchers chose these three as they are currently the largest distribution platforms for PC 
games (Steam), indie games (itch.io), and Android mobile games (Google Play Store). The researchers collected 54 games in total. This provides a substantial sample and additional games would yield diminishing returns for this study. 19 of the games within the final corpus were educational.

\subsection{Collecting VN Data}

A single researcher played all $54 \mathrm{VNs}$. Each VN was played for at least 10 hours or until completion if that occurred first. The researcher, to the best of their ability, attempted to get as many endings as possible if the game indicated that it had multiple endings. Additionally, cheats and walkthroughs were used when available in order to explore and uncover as much of the content and design of the VNs as possible during a play session. Furthermore, the researcher also created an illustrative mapping of the story tree as they played the game in order to help organize understanding of the structure of the story. The researchers chose to play the VNs instead of watching footage since gameplay is vastly different than watching a playthrough, providing more authentic results than video. This is also the typical approach in existing game research which generally relies on gameplay first and uses videos as secondary sources when necessary [6].

When playing the researcher identified and took note of the design features of each VN. A feature in this context is an audio or visual element of a game (there were no kinematic elements-i.e., touch feedback and motion based interactions-for any of the games in our corpus). For analysis, the researchers constructed a table of features identified from the earlier academic definitions in order to record how they mapped to the design features of each VN. The features extracted into the table were directly mention by each academic paper's definition. Each column of this table represented a specific feature. In this way, it was possible to identify how well the games fit into existing scholarly definitions, and a game and an academic definition could be compared visually by seeing which columns were empty or filled out.

After playing each game, the researcher recorded a "1" for that feature if it was present and "0" if it was not present. Additional features were also added to the table if an appropriate match did not exist. Specifically, four features were generated through playing games in the VN corpus, i.e., linear story structure, includes traditional text box, includes non-traditional text box, and on-click progression for text box. On a second pass the researchers replayed games and grouped together similar keywords/features to form final unique design feature categories, i.e., axial coding [78]. In total, there were 22 unique design features identified.

\section{RESULTS}

\subsection{Comparing Definitions of Visual Novel}

The data set of academic definitions of VNs consisted of 30 definitions, and collectively converged on a set of four design categories: Art and Aesthetic, Narrative Structure, Interaction, and Story Quality. Each VN definition incorporated one or more design feature from these categories, and 6.19 features on average out of the 22 unique features identified through analysis. See Figure 4 for each design feature and its frequency of occurrence in the academic definitions. Also see Figure 2 for the relationship of each design feature to the four design categories.

Art and Aesthetic refers to elements like imagery, graphics, and art style. Aesthetic elements are identified in $14 \mathrm{VN}$ definitions and surrounding discussion, e.g., [4, 22, 26, 28, 29, 40, 47, 49, 57, $68,71,75,77]$. The definitions varied in the way images were defined or described. For example, some described static images or illustrations [29]. Other definitions specify the use of images as backgrounds [31, 68] and character art $[4,28,31,71,77]$. Several definitions specifically identify the Japanese influence on both the art and general aesthetics of VNs, with 4 definitions stating that VNs have anime-inspired aesthetics [28, 29, 67, 71]. Specifically, Faizal [29] describes VNs as containing 


\section{Feature Distribution Across Academic Definition Categories}

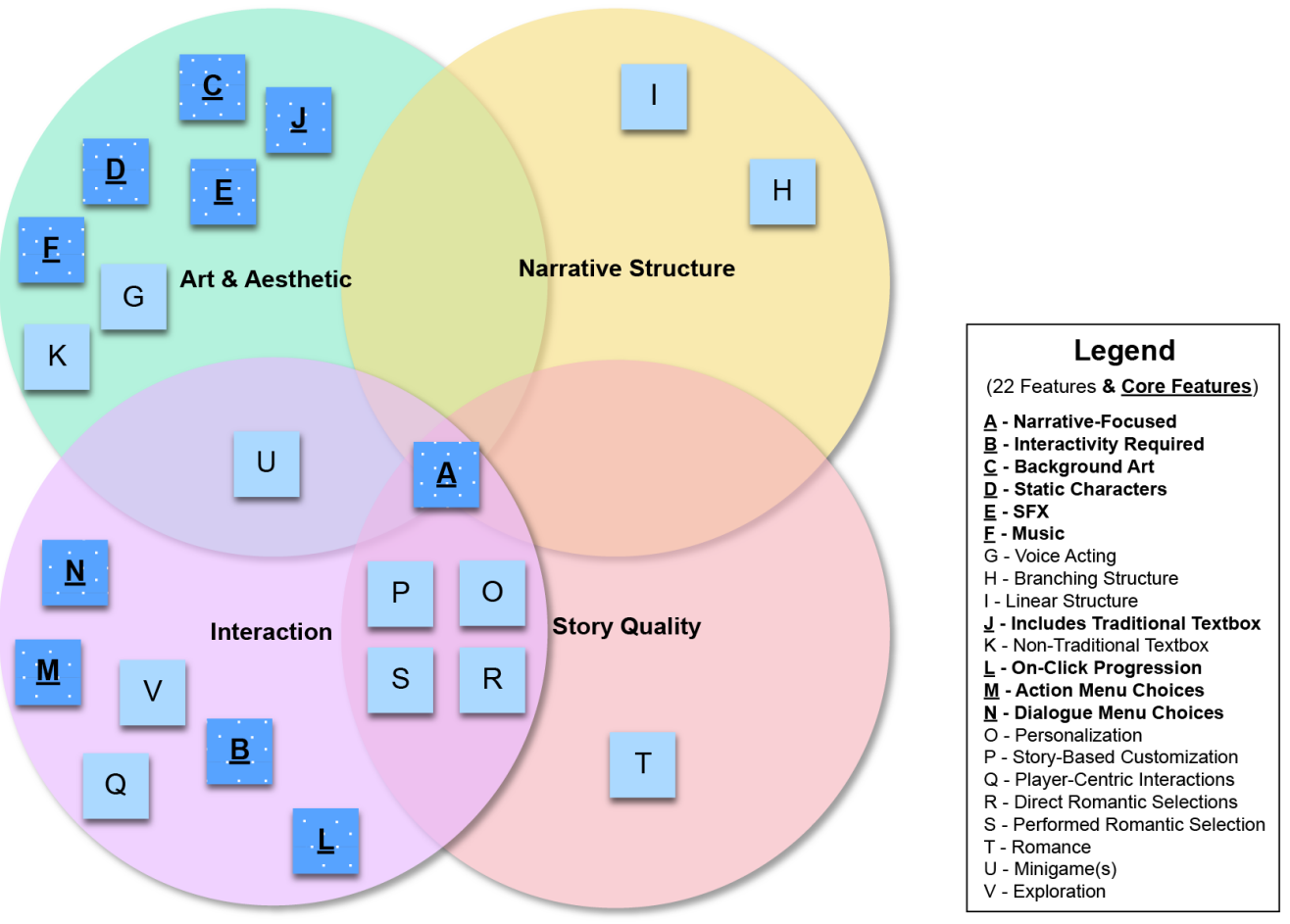

Fig. 2. All 22 features and their relationship within the 4 larger design categories identified in academic definitions.

mostly static images commonly represented in "anime cartoon" style, and Navarro-Remesal and Longuillo-Lopex's definition [67] similarly argues that VNs "derive from manga". Emi and Okuda [28] describe VNs as featuring "static graphics and anime-styled characters". While Pratama et al. [71] specify that VNs "have static backgrounds, dynamic characters that are generally manga or anime-styled and sound effects". However, while VNs did originate in Japan, they are now widely developed outside of it and convey stories outside of its cultural context, e.g. [32, 73]. Notably, a total of 9 definitions do not specify the art style of VNs [4, 26, 40, 47, 49, 57, 68, 75, 77], likely reflecting this shift.

Narrative Structure refers to structure pattern types and structural elements, and are prominently featured in 14 definitions. More specifically, narrative structure is featured in the form of specific structure patterns such as branching structures [10,22, 36, 57, 67, 71], and structural elements like endings and choices [7, 28, 29, 71, 80]. Furthermore, some definitions specified that VNs included multiple endings as a result of player choice [28, 29, 57, 80], which can indicate the presence of a branching structure to a player. This suggests that branching structures were included implicitly in more definitions than explicitly stated.

Interaction within VNs was incorporated in a variety of ways for almost all of the VN definitions (23 out of 30). Notably, some definitions specified that VNs have limited interactivity [4, 36, 40, 67] or minimal gameplay [26]. However, definitions did not agree on how limited interaction was defined or quantified, with descriptions of limited interaction varying from "limited" to "making 
decisions that affect the progress of the game" [4, 12, 40, 47, 49, 57, 67]. In terms of design elements, choices were frequently used to describe how the player might be able to mechanically interact with and impact the narrative $[4,7,10,12,28,29,40,49,50,57,67,75,80]$. More specific descriptions of interaction within the definitions identified the click-to-continue interaction, where the player clicks to receive the next part of story [7, 36, 40,57]. At least one definition identified the dialog box specifically [71], which is where story content is often delivered via click-to-continue interaction Interestingly, more complex interaction elements such as mini-games and puzzle gameplay, as seen in well-known and successful VN series like Danganronpa and Phoenix Wright, were not widely incorporated-with only one definition including RPG elements like puzzles and minigames [68].

Story Quality was identified as another significant part of VN design, with 8 definitions specifying the quality of the story itself and its elements as being an integral part of VNs. For instance, VNs have been described as "reliant on the text and the actual content instead of interactivity" and that characters play a large role in that content [12]. This is echoed by Salter et al. [79], stating that VNs are influenced by character-centric forms of storytelling and rely heavily on dialog. Moving beyond discussions of importance, there were also several notable story quality elements identified by the definitions. Cavallaro [16] highlights "plot depth and subtle characterization" as key elements of VNs while Korhonen and Halonen [49] list immersive storytelling. Romance, a popular literary genre of $\mathrm{VNs}^{6}$, was also included in 2 definitions that focused on romance and dating simulation elements $[60,79]$.

\subsection{Interactivity in Visual Novels}

Although almost all academic definitions of VNs incorporated interactivity in some form, the wide variety of ways it was employed warranted further exploration. For our study the definition of interactivity was any afforded, provided, or forced action needed to progress within the game. An interactive action, in the context of our investigation has to have some meaningful cognitive load or decision making. We measured the level of interactivity based on perceived cognitive load, frequency, and physical activity of an interaction. In particular, the focus on low amounts of interactivity-and subsequent lack of more complex interaction elements such as puzzles or minigames-could be examined by measuring the frequency of interaction for each game within our game corpus (see Figure 3).

In order to determine the frequency of interaction, we set a timer for thirty minutes and replayed each of the $54 \mathrm{VNs}$. We chose the thirty minute sampling as VNs in our corpus rarely had extensive or elaborate tutorials and generally established their core loop and rhythm of gameplay early. Before deciding on the sample method, each VN was evaluated in terms of whether the first thirty minutes were subjectively similar to their overall gameplay by the researcher who played each VN extensively. On average, the first thirty minutes were concluded to be representative of the VNs overall gameplay and thus deemed to be sufficient to indicate overall gameplay interaction frequency.

As a VN was being replayed, we noted down how many interactions, such as menu choices, were provided within the given time. Afterwards, the total number of interactions per game was divided by 30 to calculate the frequency per minute. These values were then normalized to a range between 0 and 3 by dividing by the max frequency of interactions (i.e., Pastry Lovers at 2.36 interactions on average per minute) and multiplying by 3 .

Surprisingly, most VNs had less than one interaction per minute, with an average of only 0.37 interactions per minute. Furthermore, the only exceptions to this extremely limited interaction

\footnotetext{
${ }^{6}$ Romance is tagged as the fifth-most popular sub-genre of visual novels in VNStat [1], a community-ran site that identifies trends on the Visual Novel Data Base (VNDB).
} 


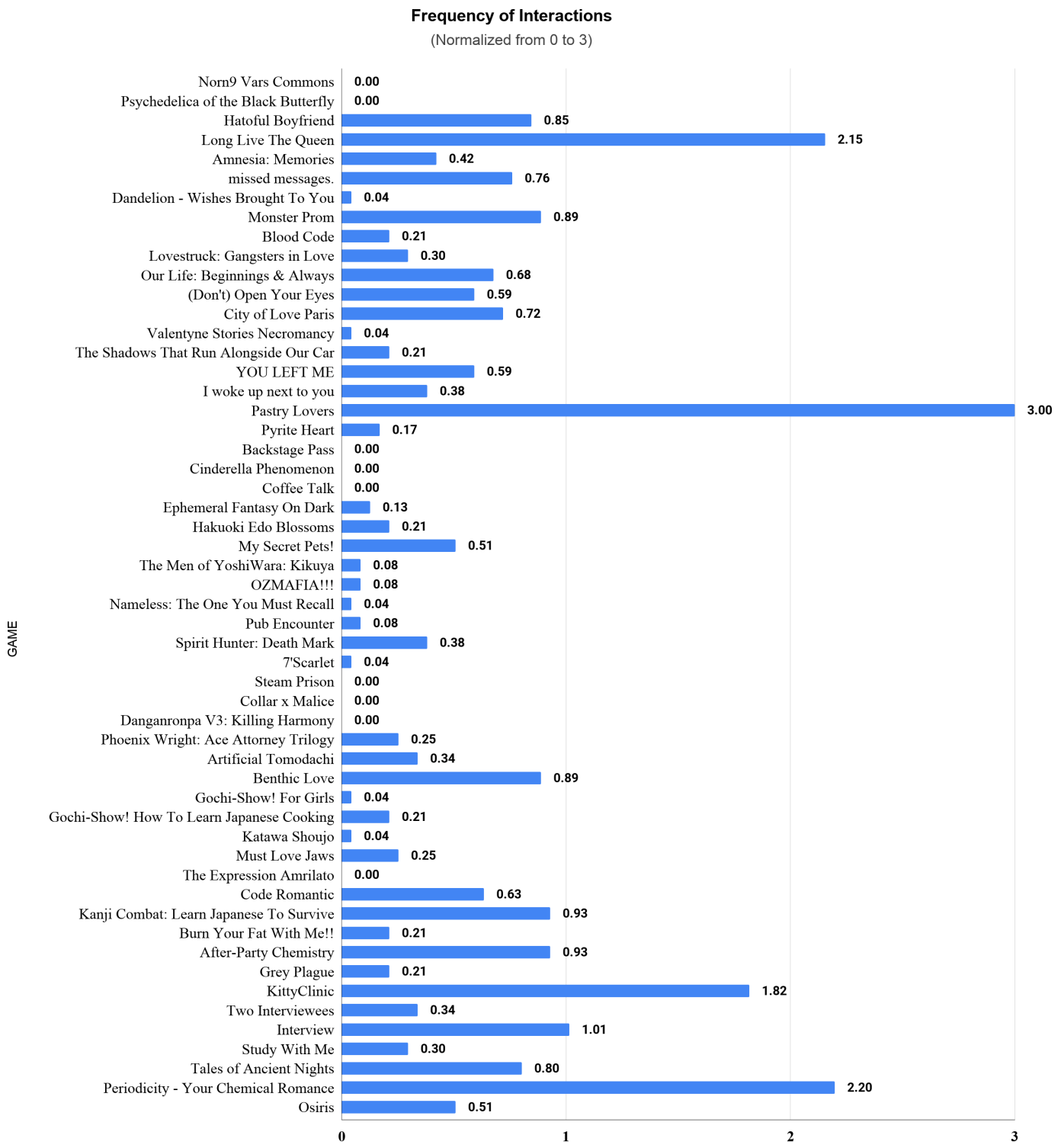

Levels of Interaction

Fig. 3. An overview of the frequency of interactions per minute within the first 30 minutes of gameplay for each of the VNs. Values were normalized to fit the range 0 to 3.

frequency still had less than three total interactions per minute. Overall, the ranges for number of interactions per game varied quite substantially. A total of 9 games had 0 interactions, due to a heavy focus on narrative, whereas stat-building VNs like Long Live the Queen and Pastry Lovers had 51 and 71 interactions respectively. 


\subsection{Crafting a Unified Definition}

In order to craft a unified definition for VNs, we first determined the set of essential core features that would be incorporated into the final definition. Breakdown per feature can be see in Figure 5. A core feature was identified as applying to at least $95 \%$ of the VNs included in the corpus. We chose $95 \%$ as the cutoff point since it gave us a high degree of confidence that the corresponding design feature was highly prevalent in VNs beyond just our corpus. We identified 10 core features of VNs: Narrative-focused, Interactivity Required, Background Art, Static Characters, SFX, Music, Includes Traditional Text Box, On-Click Progression, Action Menu Choices, and Dialogue Menu Choices.

Using these core factors, and analysis of the $\mathrm{VN}$ corpus, we propose the following new definition of a VN:

A Visual Novel (VN) is a digital narrative focused game that requires interactions where the player must be able to impact the story world or the story's progression. The story and interactions are most commonly presented through a text box and often employ additional forms of interaction including menu choices-which often contain sets of actions that the player character can perform-or dialogue options representing the player character's speech or thoughts. Crucially, VNs have On-Click Progression, where the player clicks, taps or presses a button to see the next part of the story. The aesthetics of VNs are most often conveyed through static images of characters, background art, sound effects (SFX) feedback, and soundtracks.

Previously existing academic definitions of VNs included an average of 2.17 explicit mentions of the 10 core features. The most comprehensive existing $\mathrm{VN}$ definition, by Lu [57], incorporated the highest number of core features with 7 out of 10 features included. Overall, this demonstrates that our unified definition of VNs is far more comprehensive than existing academic definitions, and provides a number of clear, identifiable features for the design of VNs. This definition also accounts for visual novels in contexts beyond entertainment, such as education, and holds true for the 19 educational visual novels within our corpus.

\section{DISCUSSION}

\subsection{Design Implications}

Our findings show that there is some mismatch in terms of emphasis between academic definitions of VNs and existing work categorized as VNs. Some academic definitions in our sample specified that VNs are interactive but overall focused more on the quantity of interaction rather than the design features enabling it.

VNs have an inherent set of interaction affordances that manifest as a result of their use of on-click progression of story content presented via text boxes and menu choice selection. While the majority of academic definitions in our sample did not include features like text boxes and on-click progression, these features were observed in all the games in our corpus. Likewise, 53 out of 54 games had menu choices presented with an onscreen menu, but these were not present in the vast majority of definitions collected for this study. The notable exception here is Burn Your Fat With Me!! [43] which did not have any choices presented to the player but did ask them to follow along with exercises presented in the game in the real world. These interaction affordances make up core design patterns or game architecture [8] of VNs. Use of these patterns allows players to "experience 


\section{Definitions Mapped By Features \\ (from a sample of 30 academic definitions)}

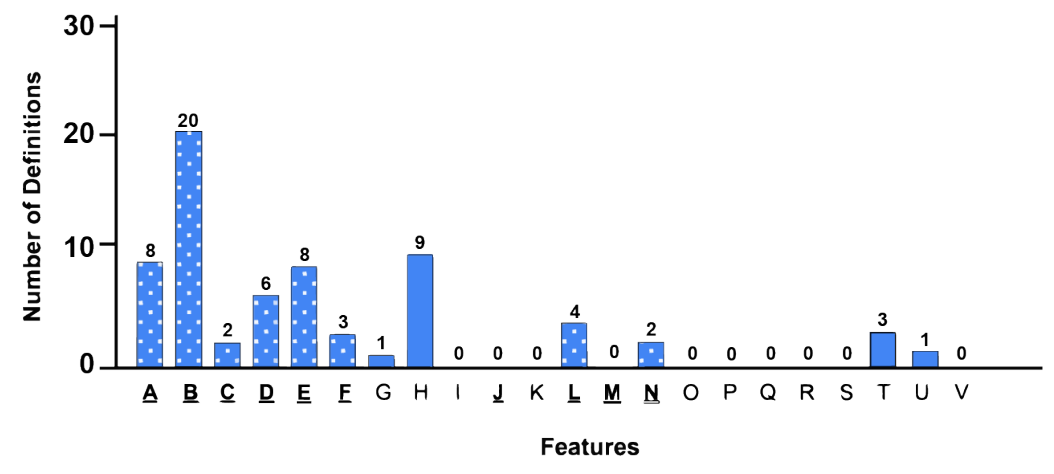

\begin{tabular}{|c|}
\hline $\begin{array}{c}\text { Legend } \\
\text { (22 Features \& } \underline{\text { Core Features })}\end{array}$ \\
\hline 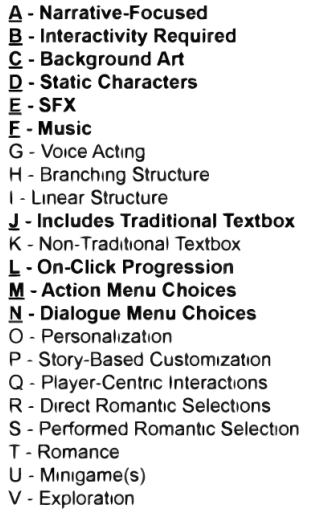 \\
\hline
\end{tabular}

Fig. 4. All 22 features mapped by how many academic definitions they appeared in from the 30 collected academic definitions.

\section{Games Mapped By Features}

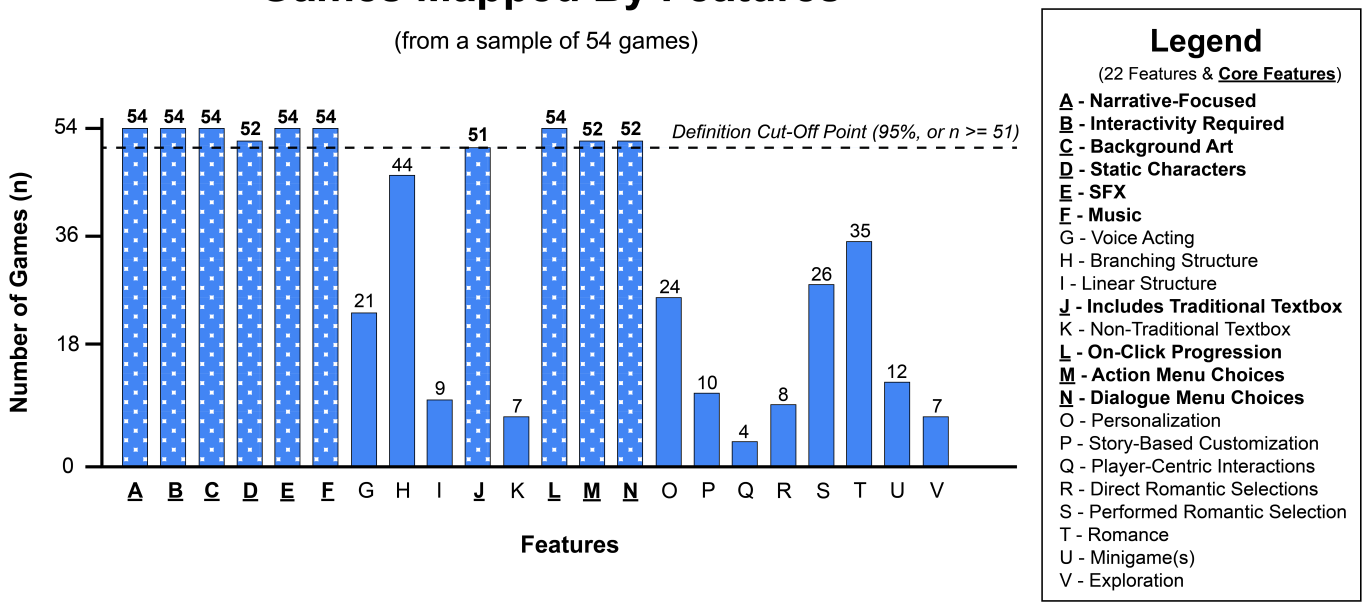

Fig. 5. All 54 games mapped by feature, across all 22 features identified in the study. Core features were identified as being present in $95 \%$ of the games in the corpus, represented by a cut-off line in the figure. The core features are identified by a dotted pattern in the figure. All values were scaled by a factor so that all 54 games were visible.

variety while remaining within the scope of a familiar and appreciated game experience for which they are already competent" [8]. In other words implementing the features players expect to be present for the genre, such as the core features identified in our study, improves the usability of the game and aligns with a player's game literacy. Not including the core features might confuse players and violate their expectations. 
The affordances provided by VNs combined with limited interaction frequency supports the genre's ability to deliver educational content. On average VNs within the corpus had 0.37 interactions per minute within the first thirty minutes of game play. Recall that levels of interactivity are measured based on perceived cognitive load. Common interactions in VNs, such as click to continue progression, are relatively simple and require limited input from the player [13]. This reduces the need for players to spend excessive cognitive load to play the game. Players can instead cognitively engage in understanding material instead of engaging in learning new mechanics.

Story structures, like branching and linear structure patterns, are often used to describe the nature of the influence the player has when interacting with a story. Branching structures allow for more variety in experience than linear structures- by allowing the player more opportunities to influence and shape the story and its outcomes through branching choices. Notably, 9 of 30 definitions in our sample included a requirement that VNs contain branching stories. However, while branching structure was heavily utilized by VNs in our corpus, linear structures were also notably prevalent, where 10 out of 54 or about $18 \%$ of the corpus did not include a branching story structure. Most notably popular games such as Phoenix Wright: Ace Attorney Trilogy [14] and Danganronpa V3: Killing Harmony [82] had a perceived linear story structure. Therefore the definition of $\mathrm{VN}$ presented by this paper does not include the restriction of specifying a particular structure type for VNs, instead leaving that design decision flexible to the designer and goals of the $\mathrm{VN}$.

\subsection{Improving the Design of Educational VNs}

One benefit of having a unified definition for VNs is that it more clearly outlines the potential design space for such games. Notably, this also allows us to examine designs within important subgenres such as educational VNs. Of the 54 VNs from the corpus, 19 were focused on educational applications. In this subsection, we analyze these 19 educational VNs with respect to our unified definition as a test-highlighting the efficacy of our definition for identifying underexplored design features and potentially improving the design of future educational VNs.

These VNs shared many common design decisions with their entertainment focused counterparts including personalization options for the player (e.g., choosing their gender or changing the player character's name) [25, 45, 45, 89, 92], having romantic love interests [43-45, 72, 84, 86, 89, 92], minigames [25, 37, 43, 72], and branching storylines with multiple endings [44, 45, 84, 86, 89, 92] However, there were some notable differences between the two where educational VNs often utilized less of the design space within the VN genre-resulting in a number of features that could potentially improve their designs and learning outcomes.

For instance, none of the educational VNs employed any story-based customization-i.e., choices that alter the player's character and affect the story. However, this is seen commonly in entertainment VNs that use stats such as Dandelion - Wishes Brought To You [85] and Monster Prom [32]. In both of these non-educational games, the player chooses activities for the protagonist which increase or decrease certain stats. These stats then alter the route of the story and possible choices presented to the player. In contrast, educational games relied more on menu choices as the main form of interacting with the story. While menu choices are simple, clear, and a direct way to communicate with players, story-based customizations have the potential to better represent effects of long term decision making. For example, in Dandelion - Wishes Brought To You every activity has a trade off. If the player chooses to do homework their character's art skills increase but their character's stress also increases. This models the idea of a work-life balance, since the player will reach a game over state if they don't carefully manage all stats, and presents it in a more intuitive form than possible with standard menu choices. This type of day planning could possibly 
be used to enhance educational games with stats focused topics such as nutrition, infection rates, or ecosystems to name a few potential examples.

Similarly, none of the educational VNs featured player-centric interactions-i.e., interactions that assist the player but ultimately do not affect the story-while a number of the entertainment focused VNs did. For instance, Hakuoki: Edo Blossoms [42] and Valentyne Stories Necromancy [9], had a glossary or journal that included important key words for the player to reference. It served as a way to explain and give context pertaining to the story for the player. Educational visual novels could benefit from implementing similar features. Just as the glossary provides context in games such as Hakuoki: Edo Blossoms, a glossary in an educational VN could remind players of previously taught concepts. For example, the game After-Party Chemistry[92] assumes that the player already knows basic chemistry and attempts to add on to that knowledge. A glossary in this case would help the player understand and remember new terminology or recall terminology they may have forgotten.

\section{LIMITATIONS AND FUTURE WORK}

When identifying features from the definitions from academic papers, three keywords were judged either too broad or not sufficiently defined to be applicable during the analysis process, and thus were excluded. These three keywords were "limited to reading," "complex stories," and "minimal gameplay". While broad they represent constructs relating to both interactivity and content complexity. The former was at least partially incorporated via analysis of frequency of interaction, while content complexity of the story itself was deemed outside the scope of the work.

It is worth noting that while a significantly sized corpus, 54 games might not be fully representative of the entire genre. Future work should aim to improve methods of constructing representative sets of games for genre construction and games research more broadly, so that the field can more effectively study and contextualize its findings.

In addition, certain features identified in the study warrant further exploration. One example is story structure. Story structure, in the context of this work, was described in terms of how it was perceived by a single researcher. Having more than one researcher play and document the games would introduce variation in descriptions that could be of interest. Further, there are more structure types and patterns that have been identified in the literature than strictly branching and linear story structures, such as fold-back and hidden story structures [15], which might have further impact on $\mathrm{VN}$ experiences.

\section{CONCLUSION}

The Visual Novel (VN) is a widely popular and recognizable narrative-focused game genre whose popularity has been steadily increasing in recent years. Their popularity has been bolstered even further with the increase of free VN game engines, such as Ren'Py [18], which have made VN creation more accessible to novice designers while allowing expert designers to construct complex and engaging work. In addition, the accessibility of VNs to a variety of players and skill levels has led to their use in high-impact domains like education [4, 13, 23, 26, 29, 31, 35, 40, 49, 51, 68, 87] and health [77, 93]. Accessibility for players is key in such domains to reach as many individuals as possible, and its popularity and narrative focus make it a highly suitable choice of gameplay for a variety of domains. Thus, the value of studying and improving VN design within HCI and Game Studies is significant. While existing academic work on VNs has provided a variety of definitions of VNs, these definitions can vary wildly-which in turn has substantial implications for the design of such games. Creating a unified definition of VNs as a genre and understanding the key elements of a VN's design allows us to better inform design and research of VNs, not just for entertainment but also their application in high impact domains such as education and health. In this paper, we 
propose a unified definition of VNs developed from analyzing existing definitions of VNs as a genre and a large corpus of commercial and educational VNs. We discussed key features from our unified definition and their implications on the design of VNs for both entertainment and serious applications.

\section{REFERENCES}

[1] [n.d.]. Romance. https://vnstat.net/tag/96

[2] Espen Aarseth. 2004. Genre trouble. Electronic book review 3 (2004), 1-7.

[3] Espen Aarseth, Solveig Smedstad, and Lise Sunnanå. 2003. A multidimensional typology of games.. In In Proceedings of the 2003 DiGRA International Conference: Level Up. DiGRA, Utrecht, The Netherlands. http://www.digra.org/wpcontent/uploads/digital-library/05163.52481.pdf

[4] Dr. Imam Dui Agusalim. 2012. Developing Interactive E-Learning Module of English Teaching to Support the Distance Education Program at EEPIS. IOSR fournal of Humanities and Social Science 5, 1 (2012), 28-32. https: //doi.org/10.9790/0837-0512832

[5] Sultan Alharthi, Olaa Alsaedi, Zachary Toups, Theresa Tanenbaum, and Jessica Hammer. 2018. Playing to Wait: A Taxonomy of Idle Games. 1-15. https://doi.org/10.1145/3173574.3174195

[6] Sultan A. Alharthi, Olaa Alsaedi, Zachary O. Toups, Theresa Jean Tanenbaum, and Jessica Hammer. 2018. Playing to Wait. Proceedings of the 2018 CHI Conference on Human Factors in Computing Systems (2018). https://doi.org/10.1145/ 3173574.3174195

[7] J Andrew, S Henry, A.N. Yudhisthira, Yulyani Arifin, and Syarifah Diana Permai. 2019. Analyzing the Factors that Influence Learning Experience through Game Based Learning using Visual Novel Game for Learning Pancasila. Procedia Computer Science 157 (2019), 353 - 359. https://doi.org/10.1016/j.procs.2019.08.177 The 4th International Conference on Computer Science and Computational Intelligence (ICCSCI 2019) : Enabling Collaboration to Escalate Impact of Research Results for Society.

[8] Thomas Apperley. 2006. Genre and game studies: Toward a critical approach to video game genres. Simulation \& Gaming - Simulat Gaming 37 (03 2006), 6-23. https://doi.org/10.1177/1046878105282278

[9] Mesange Arts. 2019. Valentyne Stories Necromancy. Game [Windows, macOS, Linux, Android]. Mesange Arts. In-development. Distributed on Itch.io. Last played March 2020.

[10] Hiroki Azuma. 2009. Otaku: Japan's database animals. U of Minnesota Press.

[11] Katerina Bashova and Veno Pachovski. [n.d.]. Poetry as a Visual Novel-a Multimedia Project. ([n. d.]).

[12] Luca Bruno. 2017. Interpreting/subverting the database: Character-conveyed narrative in Japanese visual novel PC games. Mutual Images fournal 3 (2017). https://doi.org/10.32926/2017.3.bru.inter

[13] Janelynn Camingue, Edward F. Melcer, and Elin Carstensdottir. 2020. A (Visual) Novel Route to Learning: A Taxonomy of Teaching Strategies in Visual Novels. In International Conference on the Foundations of Digital Games (Bugibba, Malta) (FDG '20). Association for Computing Machinery, New York, NY, USA, Article 77, 13 pages. https://doi.org/10 1145/3402942.3403004

[14] Capcom. 2019. Phoenix Wright: Ace Attorney Trilogy. Game [PC]. Capcom, Kyoto, Japan. Distributed on Steam. Last played March 2020

[15] Elin Carstensdottir, Erica Kleinman, and Magy Seif El-Nasr. 2019. Player interaction in narrative games. Proceedings of the 14th International Conference on the Foundations of Digital Games - FDG 19 (2019). https://doi.org/10.1145/3337722. 3337730

[16] Dani Cavallaro. 2009. Anime and the visual novel: narrative structure, design and play at the crossroads of animation and computer games. McFarland.

[17] Alexander Champlin. [n.d.]. Playing with Feelings. ([n. d.]).

[18] Robert Ciesla. 2019. Game Development with Ren'Py. Springer.

[19] Dormann Claire. 2016. Toward Ludic Gerontechnology: a Review of Games for Dementia Care.. In 1st International foint Conference of DiGRA and FDG (Dundee, Scotland) (DiGRA/FDG '16). https://doi.org/10.1145/3311350.3347173

[20] Rachel Clarke, Jin Lee, and Neils Clark. 2015. Why Video Game Genres Fail: A Classificatory Analysis. Games and Culture 12 (07 2015). https://doi.org/10.1177/1555412015591900

[21] David A. Clearwater. 2011. What Defines Video Game Genre? Thinking about Genre Study after the Great Divide. Loading... 5, 8 .

[22] Rebecca Crawford and Yuanyuan Chen. 2017. From hypertext to hyperdimension Neptunia: The future of VR visual novels: The potentials of new technologies for branching-path narrative games. 2017 23rd International Conference on Virtual System \& Multimedia (VSMM) (2017). https://doi.org/10.1109/vsmm.2017.8346298

[23] John Dominic S Diez and Edward F Melcer. 2020. Cookie Mania: A Serious Game for Teaching Internet Cookies to High School and College Students. In foint International Conference on Serious Games. Springer, 69-77. 
[24] Damien Djaouti, Julian Alvarez, and Jean-Pierre Jessel. 2011. Classifying Serious Games: the G/P/S model. Handbook of Research on Improving Learning and Motivation through Educational Games: Multidisciplinary Approaches (01 2011) https://doi.org/10.4018/978-1-60960-495-0.ch006

[25] Sleepy Duck. 2018. Learn Fapanese To Survive! Kanji Combat. Game [PC]. RIVER CROW STUDIO. Distributed on Steam. Last played March 2020.

[26] Nour El Mawas, Michael Bradford, Josephine Andrews, Pramod Pathak, and Cristina Hava Muntean. 2018. A Case study on 21st century skills development through a computer based maths game. In EdMedia+ Innovate Learning. Association for the Advancement of Computing in Education (AACE), 1160-1169.

[27] Christian Elverdam and Espen Aarseth. 2007. Game Classification and Game Design: Construction Through Critical Analysis. Games and Culture 2, 1 (2007). https://doi.org/10.1177/1555412006286892

[28] Keiji Emi and Shigeto Okuda. 2016. EPUB-type visual novel gives us happy math: A prototype of edutainment software on math. 2016 IEEE 5th Global Conference on Consumer Electronics (2016). https://doi.org/10.1109/gcce.2016.7800538

[29] M. Aliv Faizal. 2016. The effects of conversation-gambits visual-novel game on students english achievement and motivation. 2016 International Electronics Symposium (IES) (2016). https://doi.org/10.1109/elecsym.2016.7861054

[30] Emily Flynn-Jones. 2015. Well Played-Vol. 3, No. 2 (Seriously Weird Special Issue). (2015).

[31] Pedro Gabriel, Tsukasa Hirashima, and Hayashi Yusuke. 2018. A Serious Game for Improving Inferencing in the Presence of Foreign Language Unknown Words. International fournal of Advanced Computer Science and Applications 9, 2 (2018). https://doi.org/10.14569/ijacsa.2018.090202

[32] Beautiful Glitch. 2018. Monster Prom. Game [PC]. Those Awesome Guys. Distributed on Steam. Last played March 2020.

[33] W. Goddard and A. Muscat. 2017. Towards Genre as a Game Design Research Approach. In DiGRA Conference.

[34] Katelyn M. Grasse, Edward F. Melcer, Max Kreminski, Nick Junius, James Ryan, and Noah Wardrip-Fruin. 2021. Academical: A Choice-Based Interactive Storytelling Game for Enhancing Moral Reasoning, Knowledge, and Attitudes in Responsible Conduct of Research. In Games and Narrative: Theory and Practice. Springer International Publishing.

[35] Katelyn M. Grasse, Edward F. Melcer, Max Kreminski, Nick Junius, and Noah Wardrip-Fruin. 2021. Improving Undergraduate Attitudes Towards Responsible Conduct of Research Through an Interactive Storytelling Game. In Extended Abstracts of the 2021 CHI Conference on Human Factors in Computing Systems. Association for Computing Machinery, New York, NY, USA, Article 265, 8 pages. https://doi.org/10.1145/3411763.3451722

[36] Rizky Yuniar Hakkun, Dio Al Sabah A Z, Kholid Fathoni, Nana Ramadijanti, and Achmad Basuki. 2018. Online Visual Novel Game Framework. 2018 International Conference on Information and Communication Technology Convergence (ICTC) (2018). https://doi.org/10.1109/ictc.2018.8539388

[37] Angela He. 2017. Grey Plague. Game [PC]. Angela He, Distributed on itch.io. Last played March 2020.

[38] Stephanie Heintz and Effie Lai-Chong Law. 2015. The Game Genre Map: A Revised Game Classification (CHI PLAY '15). 175-184. https://doi.org/10.1145/2793107.2793123

[39] Laura Hudson. [n.d.]. Gay Dating Simulator 'Dream Daddy' Might Just Be the Gaming Miracle of the Year. https: //www.wired.com/story/dream-daddy-game-review/

[40] Duy Huynh, Phuc Luong, Hiroyuki Iida, and Razvan Beuran. 2017. Design and Evaluation of a Cybersecurity Awareness Training Game. In Entertainment Computing - ICEC 2017, Nagisa Munekata, Itsuki Kunita, and Junichi Hoshino (Eds.) Springer International Publishing, Cham, 183-188.

[41] Design Factory Idea Factory. 2015. Amnesia: Memories. Game [PC, PS Vita]. Idea Factory International. Distributed on Steam and PlayStation Store. Last played March 2020.

[42] Design Factory Idea Factory. 2018. Hakuoki: Edo Blossoms. Game [PC, PS Vita]. Idea Factory International. Distributed on Steam and PlayStation Store. Last played March 2020.

[43] Creative Freaks Inc. 2016. Burn Your Fat With Me!! Game [Android]. Creative Freaks Inc. Last played March 2020.

[44] Creative Freaks Inc. 2016. Gochi-Show! for Girls -How To Learn fapanese Cooking Game. Game [PC]. Creative Freaks Inc. Distributed on Steam. Last played March 2020.

[45] Creative Freaks Inc. 2016. Gochi-Show! How to Learn fapanese Cooking. Game [PC]. Creative Freaks Inc. Distributed on Steam. Last played March 2020.

[46] Experience Inc. 2019. Spirit Hunter: Death Mark. Game [PC]. Aksys Games. Distributed on Steam. Last played March 2020.

[47] Jaana Kemppainen, Tanja Korhonen, and Teija Ravelin. 2014. Developing Health Games requires multidisciplinary expertise.

[48] Jan Klabbers. 2003. The gaming landscape: A taxonomy for classifying games and simulations. In Digital Games Research Conference 2003.

[49] Tanja Korhonen and Raija Halonen. 2017. On the Development of Serious Games in the Health Sector - A Case Study of a Serious Game Tool to Improve Life Management Skills in the Young. Proceedings of the 19th International Conference on Enterprise Information Systems (2017). https://doi.org/10.5220/0006331001350142 
[50] Tanja Korhonen, Raija Halonen, Teija Ravelin, Jaana Kemppainen, and Kyösti Koskela. 2017. A Multidisciplinary Approach To Serious Game Development In The Health Sector.. In MCIS. 30.

[51] Tanja Korhonen, Teija Ravelin, and Raija Halonen. 2019. Development of a serious game as a method to support youth work: A case study. In GamiFIN.

[52] Raul Lara-Cabrera and David Camacho. 2018. A taxonomy and state of the art revision on Affective Games. Future Generation Computer Systems (01 2018). https://doi.org/10.1016/j.future.2017.12.056

[53] Richard Lee and Gabriel Chia. 2015. Surfacing Intuitions Through Visual Novels. In Disciplinary Intuitions and the Design of Learning Environments. Springer, 145-153.

[54] Jonathan Lessard. 2014. Game genres and high-level design pattern formations. In Proceedings of the 2014 Foundations of Digital Games Conference. Florida.

[55] Xiaozhou Li and Boyang Zhang. 2020. A Preliminary Network Analysis on Steam Game Tags: Another Way of Understanding Game Genres. In Proceedings of the 23rd International Conference on Academic Mindtrek. 65-73. https: //doi.org/10.1145/3377290.3377300

[56] Rafael P. De Lope and Nuria Medina-Medina. 2016. A comprehensive taxonomy for serious games. fournal of Educational Computing Research 55, 5 (2016), 629-672. https://doi.org/10.1177/0735633116681301

[57] Brian Lu. 2014. Hikikomori: The Need to Belong and the Activation of Narrative Collective-Assimilation through Visual Novels. Journal of Interpersonal Relations, Intergroup Relations and Identity 7 (2014), 50-61.

[58] Samuel Lynch, Karsten Pedersen, Fred Charles, and Charlie Hargood. 2019. M22 - A Modern Visual Novel Framework. In Proceedings of the 8th International Workshop on Narrative and Hypertext (Hof, Germany) (NHT'19). Association for Computing Machinery, New York, NY, USA, 9-13. https://doi.org/10.1145/3345511.3349284

[59] Simon Mccallum and Costas Boletsis. 2013. Games for Health. Chapter A Taxonomy of Serious Games for Dementia, 219-232. https://doi.org/10.1007/978-3-658-02897-8_17

[60] Heidi McDonald. 2015. Romance in Games: What It Is, How It Is, and How Developers Can Improve It. QED: A fournal in GLBTQ Worldmaking 2, 2 (2015), 32-63. http://www.jstor.org/stable/10.14321/qed.2.2.0032

[61] Edward Melcer and Katherine Isbister. 2016. Bridging the Physical Divide: A Design Framework for Embodied Learning Games and Simulations. In Proceedings of the 2016 CHI Conference Extended Abstracts on Human Factors in Computing Systems (CHI '16). https://doi.org/10.1145/2851581.2892455

[62] Edward F. Melcer, Katelyn M. Grasse, James Ryan, Nick Junius, Max Kreminski, Dietrich Squinkifer, Brent Hill, and Noah Wardrip-Fruin. 2020. Getting Academical: A Choice-Based Interactive Storytelling Game for Teaching Responsible Conduct of Research. In International Conference on the Foundations of Digital Games (Bugibba, Malta) (FDG '20). Association for Computing Machinery, New York, NY, USA, Article 78, 12 pages. https://doi.org/10.1145/3402942. 3403005

[63] Edward F Melcer and Katherine Isbister. 2021. Learning with the Body: A Design Framework for Embodied Learning Games and Simulations. In Software Engineering Perspectives in Computer Game Development. Chapman and Hall/CRC, 161-195.

[64] Edward F. Melcer, James Ryan, Nick Junius, Max Kreminski, Dietrich Squinkifer, Brent Hill, and Noah Wardrip-Fruin. 2020. Teaching Responsible Conduct of Research Through an Interactive Storytelling Game. In Extended Abstracts of the 2020 CHI Conference on Human Factors in Computing Systems (Honolulu, HI, USA) (CHI EA '20). Association for Computing Machinery, New York, NY, USA, 1-10. https://doi.org/10.1145/3334480.3382973

[65] Florian Mueller, Martin Gibbs, and Frank Vetere. 2008. Taxonomy of exertion games. In Proceedings of the 20th Australasian Computer-Human Interaction Conference (OZCHI '08). 263-266. https://doi.org/10.1145/1517744.1517772

[66] Cristina Hava Muntean, Nour El Mawas, Michael Bradford, and Pramod Pathak. 2018. Investigating the impact of an immersive computer-based math game on the learning process of undergraduate students. In 2018 IEEE Frontiers in Education Conference (FIE). IEEE, 1-8.

[67] Victor Navarro-Remesal and Antonio Loriguillo-López. 2015. What Makes Gêmu Different? A Look at the Distinctive Design Traits of Japanese Video Games and Their Place in the Japanese Media Mix. fournal of Games Criticism 2 (01 2015), 1-17.

[68] S. M. S. Nugroho, A. S. Utama, M. Hariadi, U. L. Yuhana, and M. H. Purnomo. 2018. HEIRDOM: Multiple Ending Scenario Game For Mathematics Learning Using Rule-Based System. In 2018 International Conference on Computer Engineering, Network and Intelligent Multimedia (CENIM). 192-197.

[69] Otomate. 2013. Norn9 Var Commons. Game [PlayStation Portable, iOS, PlayStation Vita]. Idea Factory. Last played March 2020

[70] Dendi Pratama, Winny Gunarti, and Taufiq Akbar. 2017. Understanding visual novel as artwork of visual communication design. Mudra furnal Seni Budaya 32, 3 (2017).

[71] Dendi Pratama, Winny Gunarti widya Wardani, and Taufiq Akbar. 2018. Building Contextual Perception Through Background Illustrations in Visual Novel with Historical Theme. 
[72] prettysmart games. 2016. Code Romantic. Game [PC]. prettysmart games. In-development. Distributed on Steam and itch.io. Last played March 2020.

[73] Toge Productions. 2020. Coffee Talk. Game [PC]. Toge Productions. Distributed on Steam. Last played March 2020.

[74] P.A. Rego, Pedro Moreira, and Luís Reis. 2010. Serious Games for Rehabilitation: A survey and a classification towards a taxonomy. In 5th Iberian Conference on Information Systems and Technologies (CISTI). 1-6.

[75] Nadia Rowena, C. Leetian, Regina E. Estuar, and Michael B. Syson. 2013. Designing Mobile Educational Games on Voter"s Education: A Tale of Three Engines. International fournal of e-Education, e-Business, e-Management and e-Learning (2013).

[76] sakevisual. 2016. Backstage Pass. Game [PC]. sakevisual. Distributed on Steam. Last played March 2020.

[77] Francisco Lepe Salazar, Tatsuo Nakajima, and Todorka Alexandrova. 2013. Visual Novels: An Methodology Guideline for Pervasive Educational Games that Favors Discernment. Grid and Pervasive Computing Lecture Notes in Computer Science (2013), 234-243. https://doi.org/10.1007/978-3-642-38027-3_25

[78] Johnny Saldaña. 2015. The coding manual for qualitative researchers. Sage.

[79] Anastasia Salter, Bridget Blodgett, and Anne Sullivan. 2018. "Just because its gay?". Proceedings of the 13th International Conference on the Foundations of Digital Games - FDG 18 (2018). https://doi.org/10.1145/3235765.3235778

[80] Moechammad Sarosa, Zamah Sari, Suhari Suhari, and Hilmy Bahy Hakim. 2017. Multiplatform Visual Novel for Culture Education in a Part of Using ICT for Developing a Lifelong Learning.

[81] Braidon Schaufert. 2018. Daddy's Play. Subversion and Normativity in Dream Daddy's Queer World. Game Studies 18, 3 (2018)

[82] Ltd. Spike Chunsoft Co. 2017. Danganronpa V3: Killing Harmony. Game [PC]. Spike Chunsoft Co., Ltd. Distributed on Steam. Last played March 2020

[83] Heintz Stephanie and Lai-Chong Law Effie. 2015. Game Elements-Attributes Model: a First Step towards a Structured Comparison of Educational Games. In DiGRA '15 - Proceedings of the 2015 DiGRA International Conference. Digital Games Research Association.

[84] Four Leaf Studios. 2012. Katawa Shoujo. Game [PC]. Four Leaf Studios. Last played March 2020.

[85] Seona Lee Somi Lee Sohyeon Jeon Sujin Ri, Marcos Arroyo. 2012. Dandelion - Wishes brought to you -. Game [PC]. Cheritz. Distributed on Steam. Last played March 2020.

[86] SukeraSparo. 2019. The Expression Amrilato. Game [PC]. MangaGamer, mirai works. Distributed on Steam. Last played March 2020.

[87] Dean Sullivan and Jessica Critten. 2014. Adventures in Research: Creating a video game textbook for an information literacy course. College \& Research Libraries News 75, 10 (Jan 2014), 570-573. https://doi.org/10.5860/crln.75.10.9215

[88] Ubisoft. 2017. City of Love: Paris. Game [Android, iOS]. Ubisoft, Ubisoft Entertainment. Last played March 2020.

[89] VanadiumValor. 2019. Periodicity: Your Chemical Romance. Game [PC]. VanadiumValor, Distributed on itch.io. Last played March 2020

[90] Mauro Vanetti. 2016. Two Interviewees. Game [Windows, macOS, Linux, Android]. Mauro Vanetti.Distributed on Itch.io. Last played March 2020.

[91] D.P. Vossen. 2004. The nature and classification of games. Avante 10 (01 2004), 53-68.

[92] xxmissarichanxx. 2017. After-Party Chemistry. Game [Windows, macOS, Linux]. xxmissarichanxx, Distributed on Itchi,io. Last played March 2020.

[93] Langxuan Yin, Lazlo Ring, and Timothy Bickmore. 2012. Using an interactive visual novel to promote patient empowerment through engagement. In Proceedings of the International Conference on the Foundations of Digital Games $41-48$.

Received February 2021; revised June 2021; accepted July 2021 